ORIGINAL PAPER

\title{
ERADICATION OF DAMAGED KERATINOCYTES IN CUTANEOUS \\ LICHEN PLANUS FORMS DEMONSTRATED BY EVALUATION \\ OF EPIDERMAL AND FOLLICULAR EXPRESSION OF CK15, INDICES OF APOPTOSIS AND REGULATORY PROTEIN S100
}

Ilze Upeniece ${ }^{1}$, Valerie Groma ${ }^{2}$, Sandra Skuja ${ }^{2}$, Vinita Cauce $^{3}$

${ }^{1}$ Department of Infectology and Dermatology, Riga Stradins University, Riga, Latvia

${ }^{2}$ Laboratory of Electron Microscopy, Institute of Anatomy and Anthropology, Riga Stradins University, Riga, Latvia

${ }^{3}$ Department of Physics, Riga Stradins University, Riga, Latvia

The study of cytoskeleton arrangement and its contribution to survival of cell-tocell contacts appears to be essential for understanding of numerous cellular and tissue processes. Applying CK15, S100 labeling and TUNEL reaction to cutaneous lichen planus subtypes, we found CK15 expression in the outer and inner root sheath of hair follicles, the basal epidermal layer, and eccrine glands. Its follicular expression was decreased in nearby inflammatory infiltrates. The CK15 immunopositivity was mostly described as weak (92.3\%) for lichen planus but equally subdivided into weak, moderate and strong in lichen planopilaris $\left(\chi^{2}=32.514\right.$; $\mathrm{df}=4 ; \mathrm{p}<0.001)$. The greatly varying apoptotic index was the highest in the lichen planopilaris involving the scalp: $81.2 \pm 10.7 ; 87.8 \pm 10.7$ and $88.0 \pm 10.5$ for the basal, spinous and upper epidermal layers, respectively. S100 positive epidermal and follicular cells did not differ in the lesions demonstrated in the study groups; still immunoreactivity was more pronounced in the scalp region of lichen planopilaris. Damage of cell-to-cell contacts was confirmed by electron microscopy. Apart from immunocyte-mediated keratinocyte death, cytoskeleton-based injury and loss of cellto-cell and matrix contacts may be of great importance, leading to eradication of degrading cells and thus contributing to the pathogenesis of lichen planus.

Key words: CK15, loss of cell-to-cell contacts, apoptosis, lichen planus, scarring alopecia.

\section{Introduction}

Lichen planus (LP) is a benign inflammatory mucocutaneous disease often presenting as chronic [1]. Cutaneous LP has different clinical subtypes based on the site of involvement and morphology of the lesions. They include papular LP, hypertrophic, vesiculobullous, actinic, annular, atrophic, linear, follicular (lichen planopilaris - LPP), LP pigmentosus and LP pigmentosus-inversus forms $[2,3]$. Lichen planus is a common dermatosis characterized by pruritic, planar, purple, polygonal papules [4]. Three differ- ent types of LPP are recognized: classic LPP, Lassueur-Graham-Little-Piccardi syndrome, and frontal fibrosing alopecia. It is a disease of adults, and children are rarely affected [5]. The classic form of LPP commonly involves the vertex, but any region of the scalp can be affected. Lesions also may be present on eyebrows and on the distal parts of the body, for example, legs and pubic region [6]. Common presenting symptoms include shedding, hair loss, and pruritus. The early scalp LPP lesions are characterized by follicular violaceous erythema and acuminate keratotic plugs which are used as diagnostic criteria. 
The lesions are commonly located at the periphery of the bald zone, appearing to be patchy or diffuse $[7,8]$. Various triggers have been proposed as potential - infections, drug reactions, reactions to metal $[9,10]$, vaccinations $[11]$ and stress $[12,13]$. The role of genetic factors in the pathogenesis of primary cicatricial alopecias (PCA) including those related to autoimmune matter has been studied as well $[7,14]$.

Hair follicle cyclic lifelong remodeling activity proves the presence of its own stem cells [15]. These multipotent stem cells, called epithelial hair follicle stem cells (eHFSCs), are located in the bulge region - specifically, at the insertion site of the arrector pili muscle in the outermost layer of the outer root sheath $[14,15]-$ and contribute to repopulation of the epidermis after injury. According to the results published by Abbas et al. (2009, 2011), Kloepper et al. (2008), and Sabeti et al. (2013) [16,17,18,19] cytokeratin 15 (CK15), a stem cell marker, labels the cells in the bulge region of the human hair follicle, the outermost layer of the outer root sheath, the basal layer of the epidermis and eccrine glands, whereas other researchers found that the basal layer of normal epidermis is usually negative for CK15, and the bulge stem cells do not contribute to the interfollicular epidermis reconstitution [20]. A further controversy is added by Cotsarelis' publication reporting about the migration of the bulge cells to the interfollicular epidermis [21]. Moreover, an inflammatory cellular infiltrate, located in the bulge region and distal follicle, may become a strong contributor to deficiency of hair follicle stem cells in LPP, explaining the permanent loss of hair (cicatricial alopecia) occurring in LPP [22]. Mobini et al. suggested that damage of eHFSCs evidenced by diminished or absent immunostaining of CK15 and mediated by cytotoxic CD8 positive cells plays a role in the pathogenesis of LPP [23]. However, the evidence that loss of eHFSCs really does occur in human PCA is poor $[24,25,26]$. Recent findings suggest that the loss of CK15-positive cells occurs in the hair follicles surrounded by perifollicular lymphocytes, while uninvolved follicles usually retain expression of CK15 [27].

The apoptotic process mediated by CD8 positive cytotoxic $\mathrm{T}$ lymphocytes and natural killers follows either the perforin/granzyme pathway or Fas/Fas ligand pathway, when cytotoxic proteins are launched to perform disseminated keratinocyte death $[28,29$, $30,31,32,33,34$ ]. However, cell death may also be induced by other mechanisms than those initiated by $\mathrm{T}$ cells, such as loss of cell-to-cell or cell-to-matrix contacts $[34,35]$. Nonspecific responses related to these mechanisms are indicated by damage of the basement membrane, which underlies and supports keratinocytes, action of metalloproteinases, etc. [7]. By light microscopy the apoptotic keratinocytes are evidenced as eosinophilic ovoid bodies found in the epidermis and subepidermal papillary regions in LP patients [36]. Colloid bodies (CB), also known as Civatte bodies, hyaline or cytoid bodies, reflecting apoptosis of keratinocytes and attenuating changes of intermediate filaments, were described several decades ago by researchers conducting electron microscopy investigations and TUNEL reaction analysis [37, 38, 39, 40, 41, 42]. However, little is known about the details of cell death when various clinical forms of LP are compared.

S100 proteins comprise a family of more than 25 different regulatory proteins, characterized by tissue-specific expression $[43,44]$. Acting as paracrine and autocrine mediators, these have been shown to be involved in numerous intracellular and extracellular functions, such as regulation of cell proliferation and survival or, alternatively, apoptosis, chemotaxis, inflammation, immune responses, $\mathrm{Ca}^{2+}$ homeostasis, differentiation of keratinocytes and dynamics of cytoskeleton constituents $[45,46,47]$. S100 proteins are expressed in normal and diseased skin [48, 49], and the subcellular distribution of these proteins in keratinocytes is heterogeneous $[50,51]$.

S100-positive dendritic cells (DCs), involved in antigen presentation, phagocytosis, healing and repair processes, have been demonstrated to be significantly increased in LP [52]. Moreover, genetic studies conducted by Wenzel et al. demonstrated that genes which encode some $\mathrm{S} 100$ proteins as well as keratins CK5, CK15, and CK17 are differently expressed in LP lesions, in comparison with skin biopsies of healthy individuals [53].

To gain a deeper understanding of the pathogenesis of LP and development of scarring alopecia, when different clinical forms appear under the scope, we selected CK15 and S100 immunohistochemistry, TUNEL reaction and electron microscopy. This choice gives information on the ongoing apoptotic process involving rearrangement of keratinocyte cytoskeleton. Our goal was to explore a pathway of eradication of damaged keratinocytes in patients with classic LPP and LP via assessment of apoptotic indices and ultrastructural evidence of keratinocyte death, paying special attention to cytoskeleton and loss of cell-to-cell contacts, correlating these findings with an evaluation of the epidermal and follicular expression of the stem cell marker CK15 and clarifying the role of the cutaneous cells specializing in immune surveillance under exposure to LP conditions.

\section{Material and methods}

\section{Study design}

This was a retro- and prospective study of patients' medical records, biopsy descriptions, and ongoing and archived punch biopsies. Thirty-five patients (16 men and 19 women) were included in this study. The 
age range was 23-77 years (mean age $=46$ ). From them, 8 patients were retrospectively diagnosed as having classic LPP of the scalp, 7 patients had LPP of the corpus and $12 \mathrm{LP}$, whereas 8 had psoriasis vulgaris and served as positive controls. All patients were admitted and treated at the Riga $1^{\text {st }}$ Hospital Clinical Centre for Skin and Sexually Transmitted Diseases, Riga, Latvia. The search criteria were: (1) "follicular LP" or "classic LPP" localized in a) the corpus or b) the scalp, and "papular LP"; (2) "off" treatment before the biopsy. Positive controls were collected during remission follow-up. The diagnosis of LPP, LP and psoriasis by both pathology report and clinical findings was necessary to be included in this study. The investigation was approved by the Riga Stradins University Ethics Committee.

\section{Light microscopy and immunohistochemistry}

Histological sections $(4-5 \mu \mathrm{m})$ were cut from formalin-fixed, paraffin-embedded punch biopsy tissues and mounted on HistoBond+ slides (Marienfeld, Lauda-Königshofen, Germany). Consecutive sections were used as negative controls of the immunohistochemical reactions, and for hematoxylin and eosin (HE) staining to confirm the diagnosis. Evaluation of structural changes was done following Olsen's guidelines (2003) and recommendations of Tandor et al. For immunochemistry, paraffin sections were dewaxed in xylene, hydrated in a series of graded ethanols, and transferred to a methanol $/ 0.3 \%$ hydrogen peroxide solution $(30 \mathrm{~min})$. After quenching of endogenous peroxidase activity, sections were washed three times in double distilled water. Heat-induced antigen retrieval was accomplished with the sections placed in $10 \mathrm{mM}$ citrate buffer for 15 minutes in a vapor lock. After antigen retrieval, specimens were allowed to cool. Thereafter, sections were successively incubated for 12 hours $\left(2-6^{\circ} \mathrm{C}\right)$ with the primary antibodies, as recommended by the manufacturer - mouse monoclonal anti-CK15 antibody (EMD Millipore Corporation, Temecula, CA, USA, $1: 80$, clone LHK15) and mouse monoclonal anti-S100 antibody (Cell Marque, Rocklin, CA, USA, 1 : 100, clone 4C4.9) accordingly. After rinsing in PBS solution, sections were incubated with HiDef Detection Amplifier for 10 min at room temperature (RT). After consecutive rinsing, incubation with HiDef Detection HRP Polymer Detector for $10 \mathrm{~min}$ (RT) was applied. The antigen sites were then visualized with 3,3'-diaminobenzidine tetrahydrochloride $(\mathrm{DAB}+\mathrm{Chromogen}$ and $\mathrm{DAB}+-$ Substrate buffer, Cell Marque, Rocklin, CA, USA) for $10 \mathrm{~min}$. The sections were counterstained with Mayer's hematoxylin, washed with tap water, dehydrated, cleared and mounted in PERTEX (mounting medium for light microscopy, Histolab, Gothenburg, Sweden). Initially, reproduction of published immunoreactivity patterns for CK15 was used as an inter- nal positive control as recommended by Kloepper et al. (2008). Thereafter, sections from a case of melanoma and human squamous cervical carcinoma were used as positive controls for S100 and CK15 immunohistochemistry, respectively. Immunohistochemical controls, with omission of the primary antibodies or substitution of them with TRIS solution, were used as negative controls.

Sections were photographed by a Leitz DMRB bright-field microscope using a DFC 450C digital camera and a Glissando Slide Scanner (Objective Imaging Ltd., Cambridge, UK) $0.5 \mu \mathrm{m} /$ pixel resolution with $20 \times$ objective, $0.275 \mu \mathrm{m} /$ pixel resolution with $40 \times$ objective. Therefore, additional reproducible measurements (Aperio ImageScope program v12.2.2.5015) of tissue markers along with their spatial distribution were obtained.

\section{TUNEL reaction}

For detection of endonucleolytic cleavage of chromatin, characteristic of apoptosis, the terminal deoxynucleotidyl transferase-mediated deoxyuridine triphosphate nick end-labeling (TUNEL) method was applied, using the In Situ Cell Death Detection Kit, POD kit and including Enzyme solution, Label solution and Converter-POD (Roche, Mannheim, Germany).

Histological slides for quantitation of TUNEL-positive cells were deparaffinized and hydrated. Pretreatment included microwave application with slides placed in $0.01 \mathrm{M}$ citrate buffer $(5 \mathrm{~min}, 360 \mathrm{~W})$. Sections were kept in $1 \%$ BSA for $1 \mathrm{~h}$ (RT) and afterwards incubated at $37^{\circ} \mathrm{C}$ with TUNEL mix (TUNEL Enzyme solution: TUNEL label, dilution $1: 9)$ for $1 \mathrm{~h}$ in a humid chamber. After washing with TRIS buffer, sections were treated for $10 \mathrm{~min}$ with methanol/0.3\% hydrogen peroxide solution. Thereafter, sections were incubated with Converter-POD solution for $30 \mathrm{~min}$ $\left(37^{\circ} \mathrm{C}\right)$, rinsed, incubated with DAB (Cell Marque, Rocklin, CA, USA), counterstained with Mayer's hematoxylin and routinely coverslipped.

\section{Evaluation of immunohistochemical staining}

The assessment of immunostaining was performed by two independent observers blind to the clinical and pathological data. Cells that were labeled by the anti-CK15 antibody and TUNEL kit and displayed brown reaction products were considered immunopositive.

Evaluation of CK15 expression: Levels of immunopositivity were defined semiquantitatively and graded as negative $(-)$, weak $(+)$, moderate $(++)$ or strong $(+++)$ when follicular and epidermal cells within the visual microscopic field were positive at $0-10 \%, 11-50 \%$, or $>50 \%$, respectively. 
Quantitation of $\$ 100$ positive cells: The staining reaction was estimated, and S100 positive cells were determined within the visual microscopic fields related to the epidermis (magnification $400 \times$ ).

Evaluation of apoptosis marker expression: We determined the total number of apoptotic epithelial cells (TNAEC) within the visual microscopic field and compared these data with the total number of epithelial cells (TNEC) appearing within the same field. The apoptotic index (AI) was used as a comparative figure $(\mathrm{AI}=\mathrm{TNAEC} / \mathrm{TNEC} \times 100)$, as described previously [54]. AI was estimated for the basal, spinous, and overlying epidermal layers, as well as follicular localization separately.

\section{Statistical analysis}

The quantitative data were expressed as means \pm standard deviation (SD) and as medians with interquartile range (IQR), whereas categorical parameters were expressed as frequencies and percentages, after being submitted to a Kolmogorov-Smirnov test to detect any differences between samples. Dispersion analysis (ANOVA) was performed using a post hoc test with Bonferroni correction. Values of $\mathrm{p}<0.05$ were considered as significant. SPSS version 21.0 was used for statistical analysis.

\section{Electron microscopy}

Punch tissue samples obtained from LP patients with the aim of study for characterization of keratinocytes' apoptosis and cytoskeleton changes were processed for conventional transmission electron microscopy. Materials were fixed in $2.5 \%$ glutaraldehyde in $0.1 \mathrm{M}$ phosphate buffer at $\mathrm{pH} 7.4$, postfixed in $1 \% \mathrm{OsO}_{4}$, dehydrated in a series of graded ethanols and acetone, and embedded in epoxy resin. Ultrathin, 60-nm-thick fine sections were cut with an LKB ultramicrotome, collected on copper grids, double stained with uranyl acetate and lead citrate, and examined in a JEOL 1011 transmission electron microscope (JEOL, Japan) at accelerating voltage 80$100 \mathrm{kV}$ and at magnification 4000-30 000×.

\section{Results}

\section{Clinical findings}

From the medical records, data including age, gender, duration, site and extent of disease, signs and symptoms, and presence or absence of itching, were obtained. Common findings in the scalp included follicular hyperkeratosis, pruritus, areas without hair, and perifollicular erythema, whereas the majority of corpus LPP patients had pink to violaceous, shiny, pruritic polygonal papules and plaques, and follicular hyperkeratosis. Lichen planus was characterized by flat-topped, pink to violaceous, shiny, pruritic polygonal papules and plaques.

\section{Histologic findings}

The early lesions demonstrated in LPP manifested as dilated hair follicles containing keratotic plugs with underlying hypergranulosis. Lymphocytic perifollicular and perivascular inflammation was present. Epidermal and dermal histopathology in the case of LPP confirmed cellular degeneration and vacuolization in the basal epidermal layer, acuminate keratotic plugs, and lymphocytic band-like inflammation, whereas LP presented with focal hypergranulosis, basal cell layer degeneration and a band-like subepidermal infiltration (Table I). Eosinophilic epidermal and upper dermal CBs were seen.

\section{Immunohistochemistry findings}

\section{CK15 immunostaining}

Cells labeled by the anti-CK15 antibody displayed a brown cytoplasmic staining pattern. Expression of CK15 in epithelial cells was demonstrated in the outer and inner root sheath of hair follicles, the basal layer of epidermis and eccrine glands. The results describing levels of epidermal and follicular cytokeratin expression and found to be greatly varying from absence and weak to strong are summarized in Fig. 1.

Comparing the levels of CK15 expression in two subtypes of LPP and LP, when localization, namely, epidermal or follicular, was not taken into consideration (Fig. 2), we found that LP and psoriasis mostly (92.3 and $87.2 \%$, respectively) presented with weak CK15 immunopositivity, whereas both LPP types revealed almost equal splitting into the levels of weak, moderate and strong immunopositivity $\left(\chi^{2}=32.514\right.$; $\mathrm{df}=4 ; \mathrm{p}<0.001)$. Both LP forms displayed follicular CK15 immunopositivity in the hair follicle bulge region, specifically, the outermost layer of the outer root sheath, which usually was strong, and strong to moderate in the inner root sheath. Moreover, CK15 positive follicular cells revealed a remarkable diminishment when lymphocytic infiltration happened to be localized in the close vicinity (Fig. 3). Epidermal expression of CK15 was almost nil in the case of LP, whereas its positive decoration of the cytoplasm of some particular keratinocytes of the basal epidermal layer was demonstrated in LPP. Psoriatic skin samples demonstrated some discontinuous expression of CK15 in keratinocytes constituting the basal layer of epidermis mostly estimated as weak.

\section{TUNEL reaction results}

Study groups revealed greatly varying TUNEL reaction results summarized in Fig. 4.

The AI value was lower in the LP group than in the LPP group, revealing an increase in index values from the basal toward the upper epidermal layer (Fig. 5, 6). The highest estimates were demonstrated for the 
Table I. Histologic features of LPP and LP

\begin{tabular}{|c|c|c|c|}
\hline \multirow[t]{3}{*}{ FINDINGS } & \multicolumn{3}{|c|}{ Number OF CASES } \\
\hline & \multicolumn{2}{|c|}{ LPP } & \multirow[t]{2}{*}{ LP } \\
\hline & SCALP & CORPUS & \\
\hline \multicolumn{4}{|l|}{ Epidermal involvement: } \\
\hline Lichenoid reaction & 3 & 3 & 10 \\
\hline Vacuolar cellular changes & 6 & 6 & 12 \\
\hline Spongiosis & 0 & 0 & 8 \\
\hline Hypergranulosis & 0 & 1 & 7 \\
\hline Hyperplasia & 0 & 0 & 2 \\
\hline $\begin{array}{l}\text { Absence of epidermal } \\
\text { involvement }\end{array}$ & 1 & 1 & 0 \\
\hline \multicolumn{4}{|l|}{ Follicular involvement: } \\
\hline Lichenoid reaction & 4 & 4 & 3 \\
\hline Vacuolar cellular changes & 7 & 5 & 4 \\
\hline Spongiosis & 3 & 0 & 1 \\
\hline Tufted follicles & 2 & 3 & 0 \\
\hline Cysts & 2 & 3 & 0 \\
\hline $\begin{array}{l}\text { Absence of follicular } \\
\text { involvement }\end{array}$ & 0 & 0 & 0 \\
\hline \multicolumn{4}{|l|}{ Keratinization pattern: } \\
\hline Parakeratosis & 1 & 0 & 3 \\
\hline Hyperkeratosis & 2 & 1 & 5 \\
\hline Follicular plugging & 6 & 7 & 4 \\
\hline \multicolumn{4}{|l|}{ Inflammation: } \\
\hline Mild & 3 & 1 & 2 \\
\hline Moderate & 2 & 2 & 8 \\
\hline Severe & 2 & 3 & 2 \\
\hline Absent & 1 & 1 & 0 \\
\hline \multicolumn{4}{|l|}{ Localization of infiltrate: } \\
\hline Dermal & 2 & 4 & 12 \\
\hline Follicular & 6 & 4 & 0 \\
\hline Interfollicular & 0 & 0 & 0 \\
\hline \multicolumn{4}{|l|}{$\begin{array}{l}\text { Localization of fibrotic } \\
\text { changes: }\end{array}$} \\
\hline Upper dermis & 4 & 3 & 2 \\
\hline Lower dermis & 3 & 7 & 0 \\
\hline Perifollicular & 5 & 4 & 0 \\
\hline
\end{tabular}

scalp region of LPP; these were expressed as follows $-81.2 \pm 10.7 ; 87.8 \pm 10.7$ and $88.0 \pm 10.5$ for the basal, spinous and upper epithelial layers, respectively. AI dispersion analysis (ANOVA) showed statistically significant differences between patient groups within the basal $(\mathrm{F}=108.7$; $\mathrm{p}<0.001)$, spinous $(\mathrm{F}=29.6$; $\mathrm{p}<0.001)$, and upper $(\mathrm{F}=10.7 ; \mathrm{p}<0.001)$ epidermal layers. In the basal epidermal layer the mean

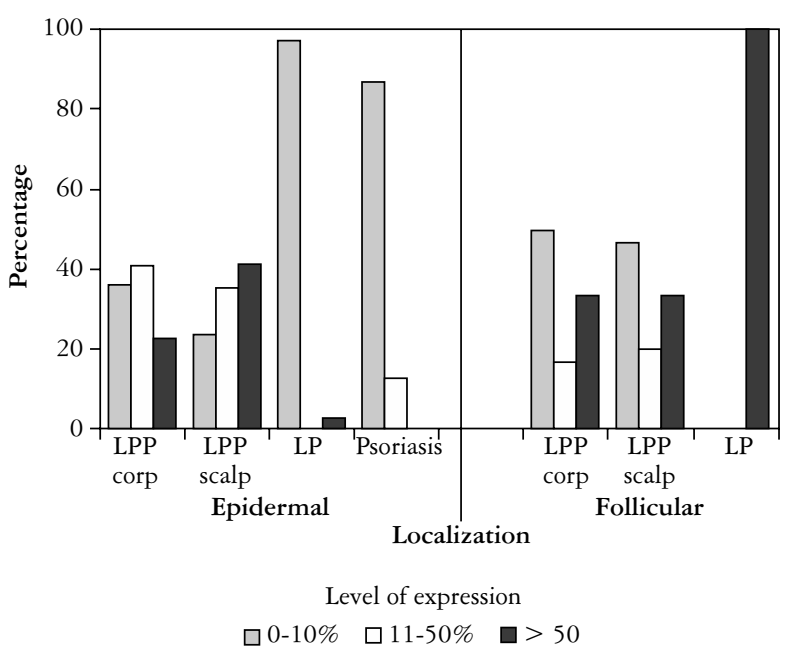

Fig. 1. Distribution of CK15 expression levels when epidermal and follicular localization is distinguished in study groups

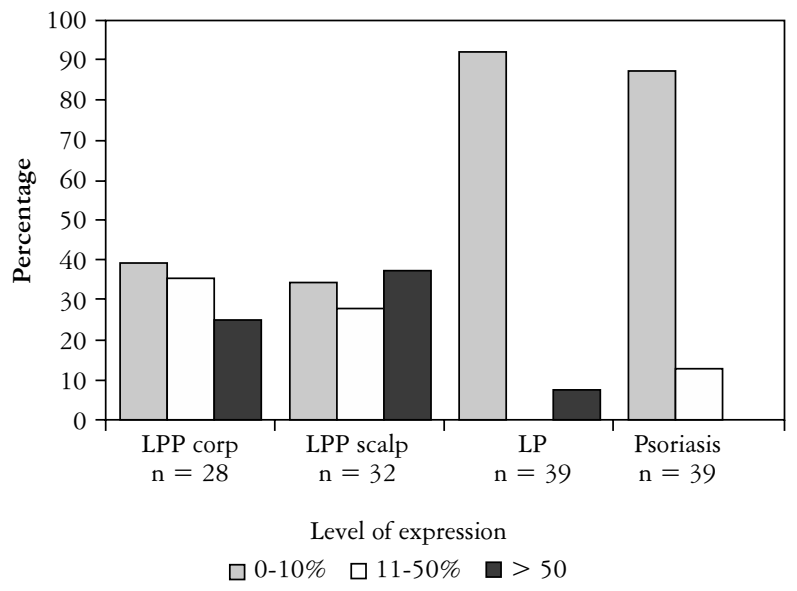

Fig. 2. Diagram complementary to Fig. 1 and summarizing distribution of CK15 expression levels in study groups

difference was 18.49 (95\% CI: 10.60-26.39), 35.91 (95\% CI: 27.36-44.46), 54.41 (95\% CI: 46.6262.20), 26.57 (95\% CI: 17.56-35.57) and 27.84 57 (95\% CI: 18.19-37.48) when LPP of corpus and scalp, LPP of corpus and LP, LPP of scalp and LP, LPP of scalp and psoriasis, and LP and psoriasis were compared, respectively, whereas in the spinous layer and upper epithelial layers these variables were observed as 21.67 (95\% CI: $12.05-31.29), 8.78$ (95\% CI: from -1.37 to 18.93 ) and 30.45 (95\% CI: $21.05-39.86$ ), and 13.02 (95\% CI: 2.91-23.13), $5.42(95 \% \mathrm{CI}$ : from -5.24 to 16.09 ), 18.44 (95\% CI: 8.56-28.32), 29.44 (95\% CI: 18.21-40.67) and 22.11 (95\% CI: 9.7-34.47), respectively. It is worth noting that apart from the damaged apoptotic keratinocytes, TUNEL positivity was demonstrated within dermal inflammatory infiltrates and intraepithelial lymphocytes, which were not specifically counted in this study. 


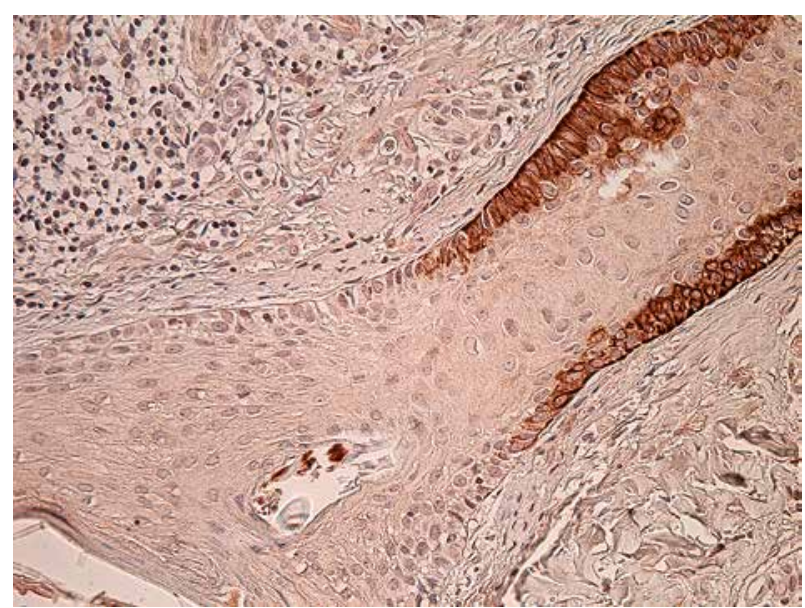

Fig. 3. Immunostaining for CK15 of a cutaneous LP form. The outermost layer of the outer root sheath is heavily decorated with the anti-CK15 antibody in the case of LPP of the corpus, but appears to be diminished close to the inflammatory infiltrate. Original magnification $250 \times$

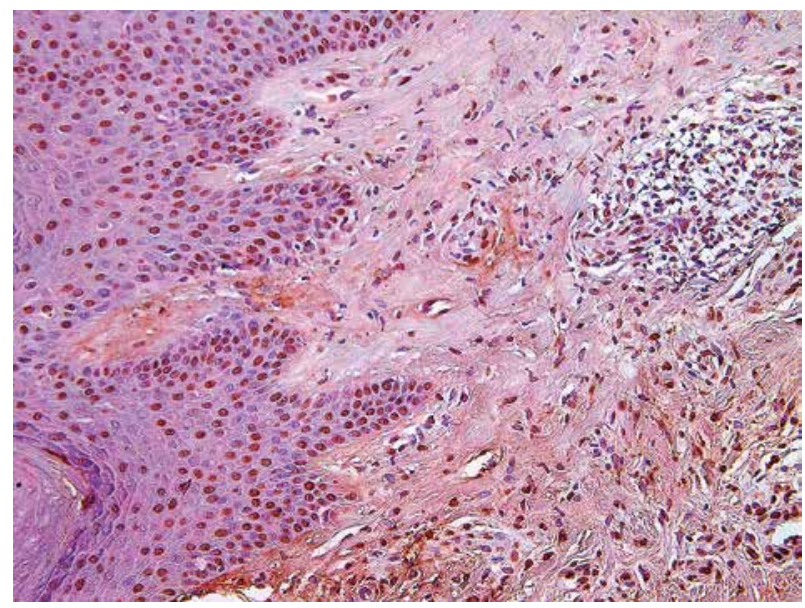

Fig. 5. TUNEL positive cells moderately decorate epidermis in LP, some inflammatory dermal cells display positivity as well. Original magnification $200 \times$

\section{S100 immunostaining}

Strong S100 cytoplasmic and, sometimes, nuclear staining was observed in the samples obtained from all patient groups. It was confined to cells localized in the spinous epidermal layer, slightly more scarcely to the suprabasal and basal layer of the epidermis. Hair follicles displayed S100 positivity confined to the outer root sheath (Fig. 7). The eccrine glands, the apocrine glands, and the sebaceous glands were all negatively stained with the anti-S100 antibody. The majority of S100-positive dermal cells presented perivascular localization. Microscopically, the lesions from the scalp region of LPP patients demonstrated stronger and denser immunoreactivity when compared to the corpus region. Still, there were no differences found in S100 positive cells in the epider-

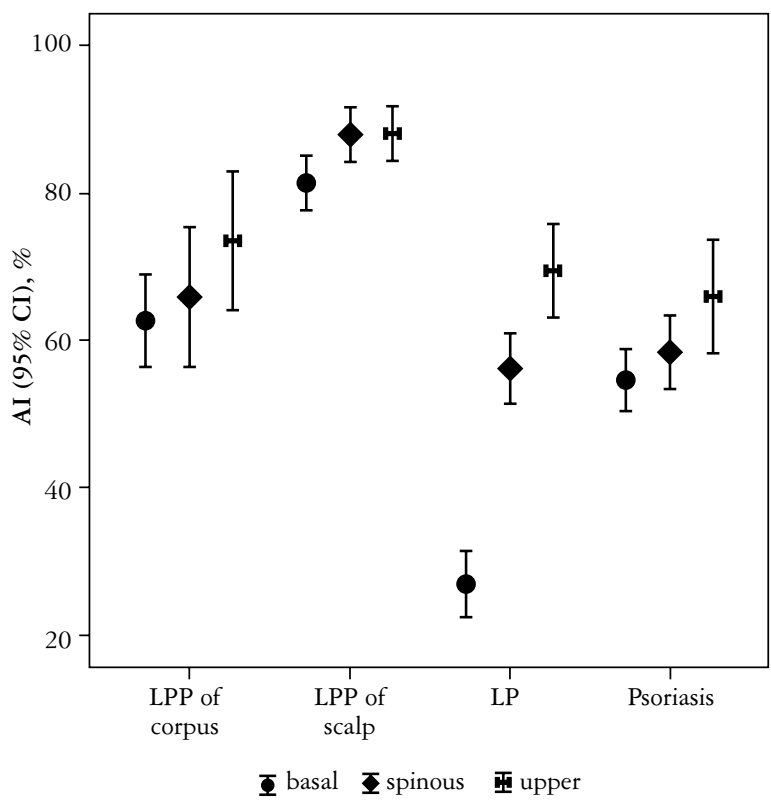

Fig. 4. Assessment of AI in the LP, LPP of the scalp, corpus region and psoriasis

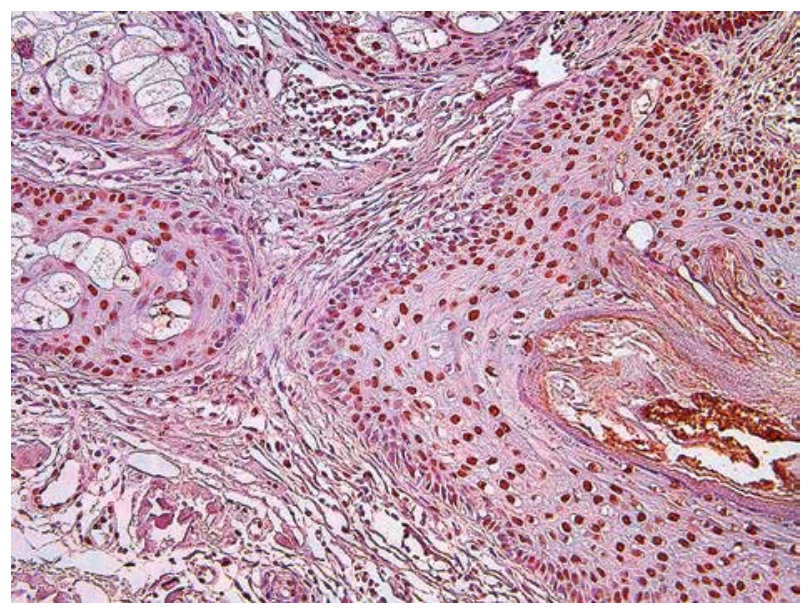

Fig. 6. Numerous TUNEL positive cells' nuclei visible in the LPP lesions affecting scalp. Original magnification $200 \times$

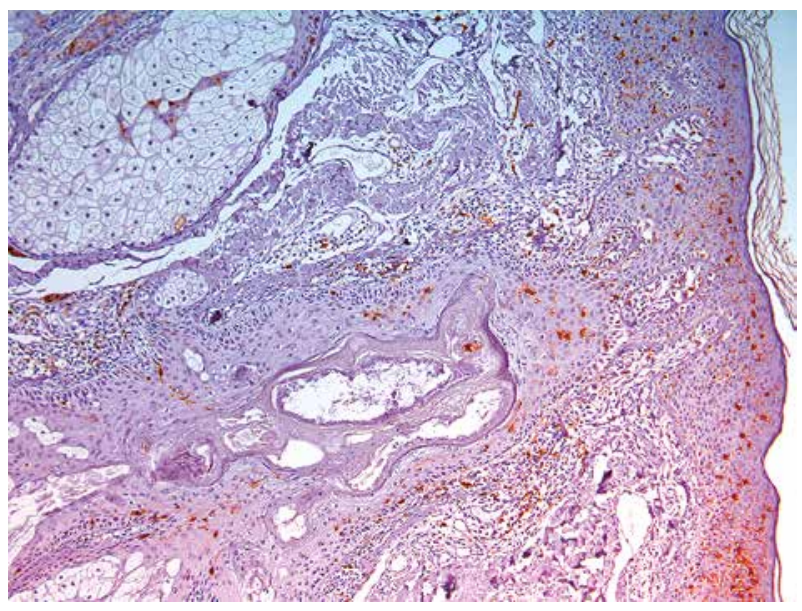

Fig. 7. S100 immunostaining in LP. Numerous S100 positive dendritic cells present in the outer root sheath and epidermis in scalp region. Original magnification $100 \times$ 


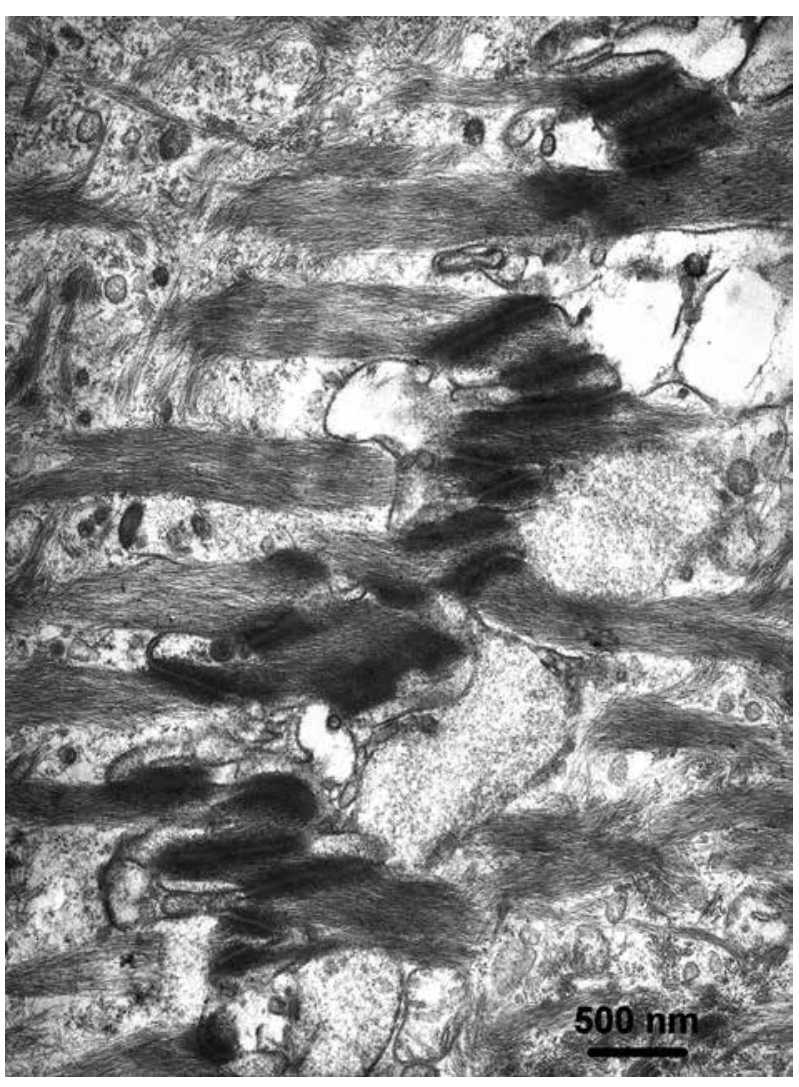

Fig. 8. Bundles of intermediate filaments attached at the cytoplasmic site to the desmosomal plaques, local widening of intercellular spaces. Original magnification $12000 \times$

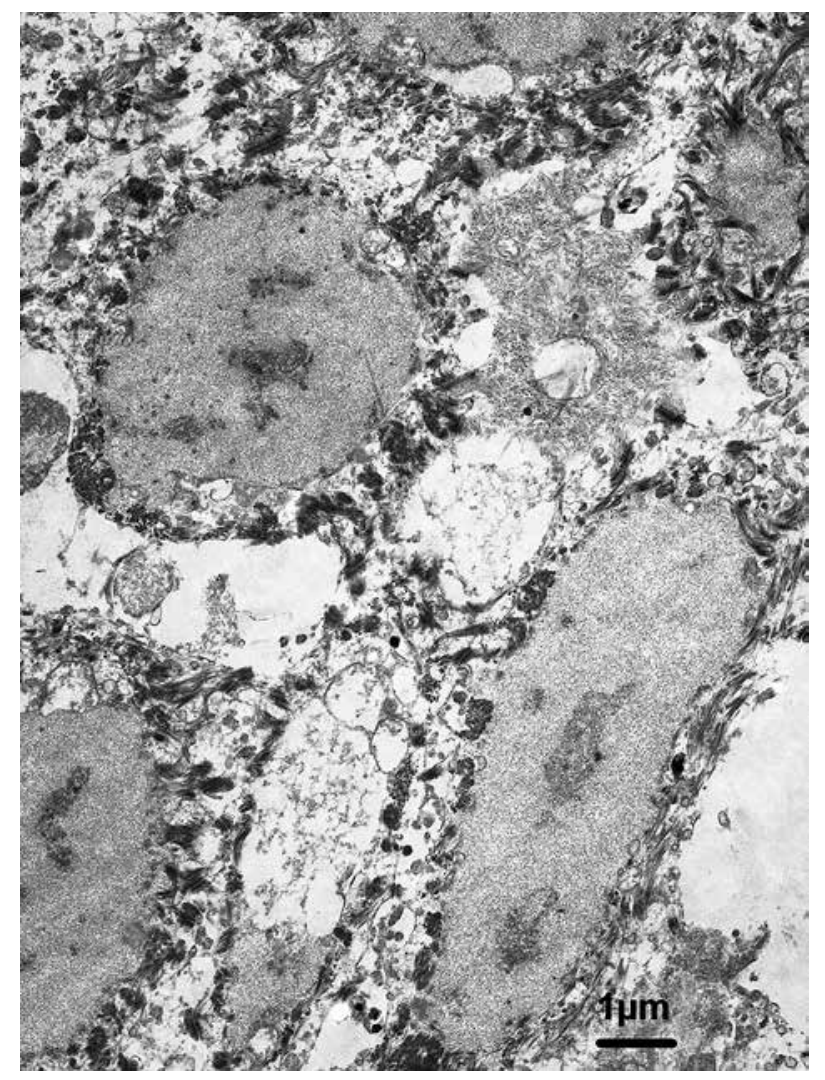

mis and hair follicles calculated for samples obtained from LP and LPP patient groups, and evaluations of S100 immunostaining were described as follows: LPP of the corpus 9.0 (IQR 6; 11.0), scalp region 4.0 (IQR 2.3; 18.8), and LP 10.5 (IQR 6.0; 14.3), whereas epidermal S100 immunostaining done on psoriatic samples was virtually nil, described as 0.0 (IQR $0.0 ; 1.0$ ), and differed significantly ( $\mathrm{p}<0.001$ ) when compared to aforementioned study groups.

\section{Electron microscopy}

Transmission electron microscopy aimed to achieve better understanding of LP pathogenesis via exploration of cellular damage and alteration of cell-to-cell or cell-to-matrix contacts accentuating the role of the keratinocyte cytoskeleton in the conditions studied. The lesions of 8 patients with LP were considered and processed for ultrastructural analysis based on immunohistochemistry findings and a punch biopsy size restriction. Keratinocytes of the basal layer often demonstrated detachment from the basement membrane and greatly varying degenerative changes. The tonofibrillar system appearing in the affected keratinocytes demonstrated a wide spectrum of changes - from thick and densely packed bundles of intermediate filaments attached locally to the desmosomal plaque (Fig. 8) and the occasional widening of the intercellular spaces up to chaotically distributed

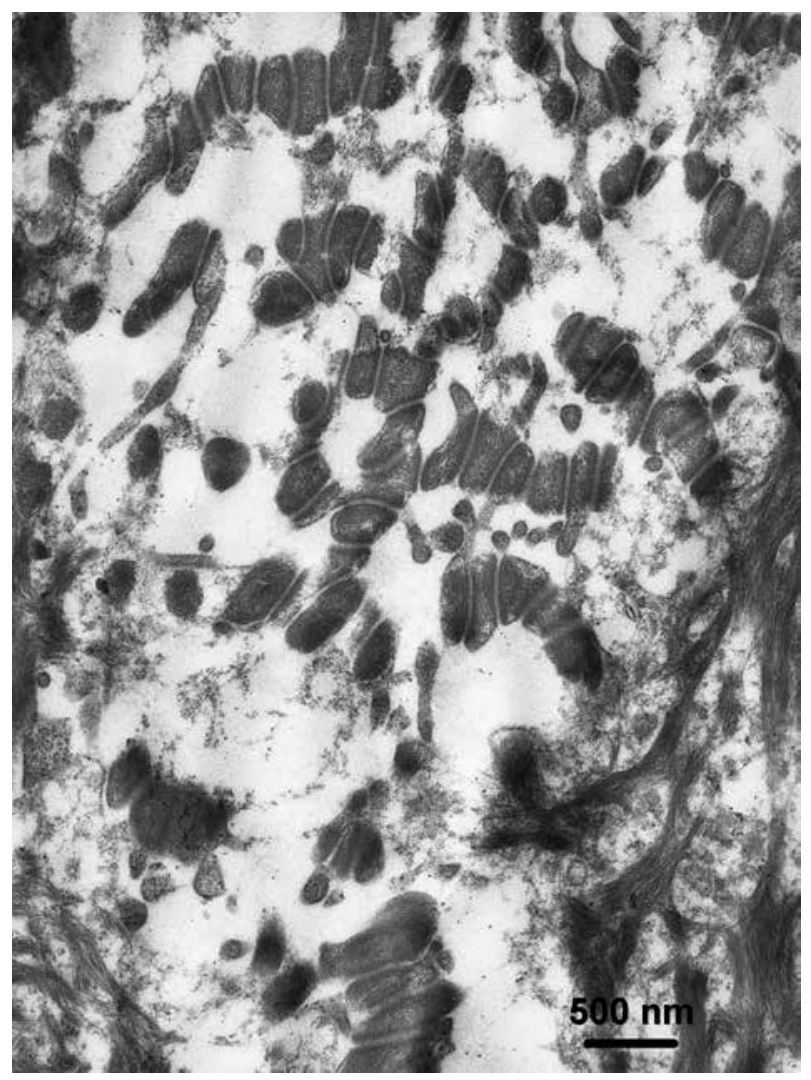

Fig. 9, 10. Severe disturbance of cell-to-cell contacts and disorganization of the keratinocytes' tonofibrillar system, appearance of free desmosomal complexes in the expanded intercellular space. Original magnification $500 \times$ and $12000 \times$ 
and thinned bundles of intermediate filaments, and even free desmosomal complexes (Fig. 9, 10) in the expanded intercellular spaces separating damaged keratinocytes with low cytoplasmic electron density. Rounded, oval and irregularly shaped accumulations of tonofilaments with sparse cytoplasmic organelles, which were assumed to be $\mathrm{CB}$, were demonstrated in or between the degenerating keratinocytes separated from the underlying basement membrane or in the upper dermis (Fig. 11). The altered basement membrane demonstrated a discontinuity with local ruptures or, conversely, multiplications. Sometimes, remnants of the basement membrane were demonstrated freely in the dermis, being surrounded by collagen fiber microfibrils.

\section{Discussion}

Lichen planus affected individuals enrolled in this study were found to be in accordance with the literature data regarding their age and a female gender predominance [1]. Patients affected by classic LPP of the corpus and LP presented with polygonal pruritic papules, whereas in the case of classic LPP of the scalp they presented with perifollicular erythema, follicular hyperkeratosis, and permanent hair loss. Apart from distribution of clinical subtypes based on the site of involvement, classic LPP of the corpus and LP shared many common clinical characteristics at the time of presentation; therefore, punch biopsy was considered.

Band-like lymphohistiocytic infiltrate at the dermal-epidermal junction and in the upper dermis, basal cell degeneration, foci with wedge-shaped hypergranulosis, slight spongiosis in the spinous layer, and lichenoid reaction of the epidermis (a jagged "sawtooth") were demonstrated in most of the LP patients recruited in this study and reported by other authors [2, 55], whereas, similarly to Poblet et al. we found dilated hair follicles, infundibular hypergranulosis lymphocytic, perifollicular fibrosis and infiltration in the isthmus and infundibulum areas of the hair follicles in the classic LPP [56]. According to the aforementioned study, the basal layer of the hair follicle contained keratinocytes severely affected by inflammatory infiltrates in the case of classic LPP; moreover, infiltrates heavily decorated the interfollicular epidermis. By contrast, the Mirmirani et al. study, based on evaluation of 20 cases of primary scarring alopecia, did not report any differences in the histopathological profile between LPP, frontal fibrosing alopecia, pseudopelade (Brocq), and central centrifugal alopecia, and suggested that characterization of hair follicles' and sebaceous glands' appearance, type, location, and extent of the inflammatory infiltrate may be of greatest value in guiding the treatment of patients with primary cicatricial alopecias [57]. Our findings suggest a milder involvement of the inter-

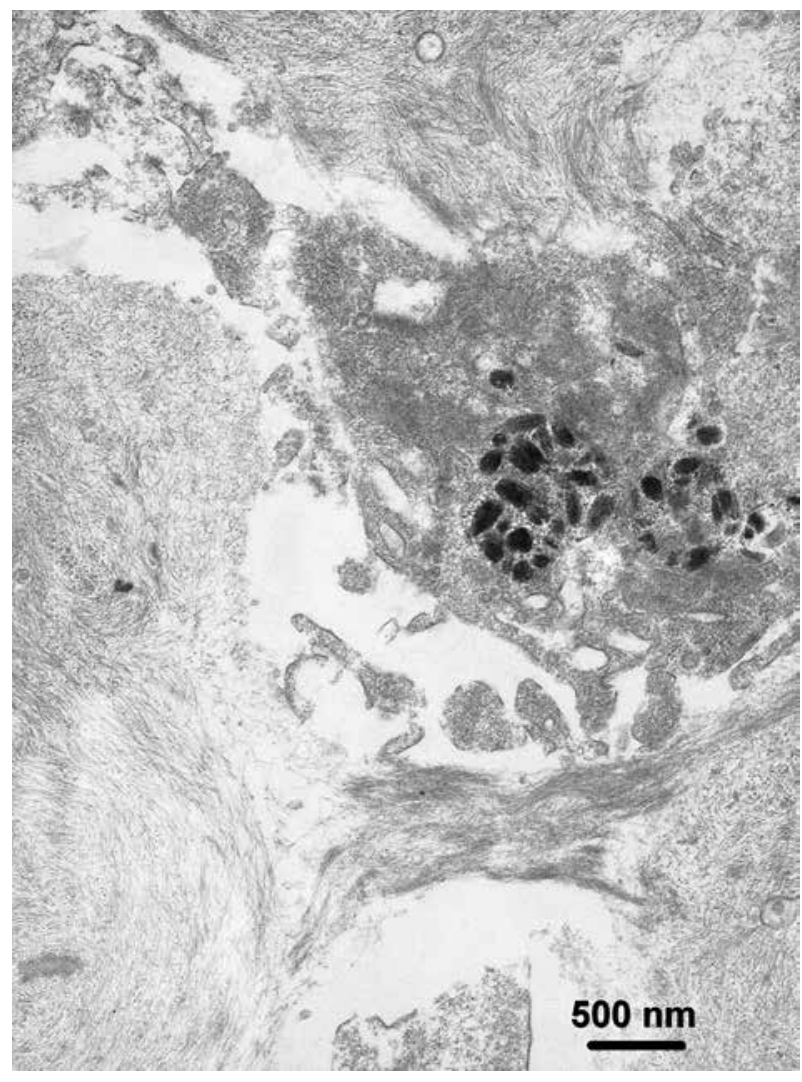

Fig. 11. Degenerating keratinocytes showing chaotically oriented tonofilaments with sparse cytoplasmic organelles. Original magnification $12000 \times$

follicular epidermis, and this is in agreement with other researchers [2]. Permanent destruction of hair follicle, followed by its substitution by fibrous tissue, is reported to be common in primary cicatricial alopecias [58]. We found moderate fibrosis at the sites of former follicles in the end stage of LPP by routine histopathology, revealing similarity of common destructive follicular changes and appearance of late fibrotic changes in the studied groups.

CK15, used in a number of studies, has been shown to be a nonspecific but still reliable marker of eHFSCs [19]. Interestingly, the comparison of both types of LPP done by us revealed a wide spectrum of levels defined for CK15 and described as weak, moderate and strong, whereas LP and psoriasis mostly presented with weak immunopositivity. Positive staining of CK15 was noted in all stages of the hair growth cycle, but CK15-positive follicular stem cells disappear in affected follicles when they enter the catagen phase, thus allowing the authors to outline the clinical relevance of the CK15 marker in LPP and explain the progression of the disease, despite the use of therapy [25, 27]. Recent studies showed CK15 staining in the outermost layer of the hair bulge of normal human scalp samples, as well as in the proximal isthmus, infundibulum, outermost layer of the 
sub-bulge, the basal interfollicular epidermis and eccrine glands [59, 60, 61]. Exploration of the literature data on development of scarring alopecia led us to the understanding that clones C8/144B and LHK15 of anti-CK15 antibodies are mostly used [62], and LHK15 antibody, recognized as a marker for bulge epithelial stem cells in humans [63], was selected for this study. Other investigators have shown that bulge cells may serve as frame builders in the follicular construction of a hair [64], and the opinion that loss of stem cells in the bulge area of the hair follicle is the reason for alopecia has been established [7, 22, 23]. CK15 has been shown to be an early predictor of keratinocyte differentiation, directing it to become either epidermal or follicular [19]. Estimating development of fibrosis, which is a common histopathologic finding in primary cicatricial alopecias, we found that CK15 negativity appears along with marked fibrosis. This statement is in agreement with previously reported data [25]. For all that, studying the relevance of CK15, we have to confirm that a suggestion made by Kloepper $e t a l$. and Inoue $e t$ al. on the usefulness of application of a panel of eHFSC markers is justified $[19,60]$. Heterogeneity of hair follicle progenitor cells reported by Hoang et al. still should be considered in such application [25]. Additionally, it is necessary to note that applying a digital immunohistochemistry approach with the purpose to objectively detect and estimate tissue markers studied, as stated in the material and methods, we obtained results comparable with the manually obtained data. Similarly, no statistical difference between the variance of performance when comparing glass and digital interpretations was found by other researchers $[65,66]$. Still, it seems that, when applying a digital approach, one should be careful to focus on specific pathology tasks to reduce sources of variability diluting findings [67], and prevent escalation of data size and complexity developing in well-designed databases [68]. In our study, the CK15 antibody stained hair follicles, where it often remained negative in the interfollicular epidermal region, and, especially, when localized next to inflammatory infiltrate. CK15 negativity was demonstrated in inflamed follicles, leading to the speculation that eHFSC loss happens due to inflammation [26]. Both resident and migratory lymphocytes abundantly colonize skin as an organ, but the question of whether and how immune cells impact skin epithelial stem cells still remains unclear. Recently published results on eHFSCs controlled by skin-resident macrophages and evidence that macrophage apoptotic death in early telogen causes activation of stem cells provide new insights on this issue [69]. Accepting that immune factors, in general, and cell-mediated immunity, in particular, play an essential role in the pathogenesis of the LP, we performed immunostaining for CD68 and CD8 phenotype and evaluated immunocytes surrounding the bulge area of the hair follicle (data not shown). We observed an increase of $\mathrm{CD} 8+$ cells along with elevation of inflammatory infiltration, confirming the contribution of $\mathrm{CD} 8+$ lymphocytes adjacent to the affected keratinocytes to the eradication of these cells. Based on a speculative approach, a suggestion regarding inflammatory or, possibly, autoimmune targeting of CK15 positive stem cells in LPP could be made. It is worth noting that in this context our results are in agreement with the hypothesis proposed by Harries et al. reporting about LPP inflammation-induced eHFSC death. According to this proposal, a unique niche created for these cells in the hair follicle bulge is destroyed, and established immune privilege collapses. Therefore, strong perifollicular lymphocyte-mediated damage of keratinocytes, and even more pronounced when accompanied by enzymatically guided remodeling and degradation of extracellular matrix occurring in LPP, suggests a role of the above-mentioned factor in the development of scarring alopecia. We propose that detection of CK15-positive cells along with evaluation of lymphocytic infiltrate impact in the infundibulo-isthmic bulge portion of the hair follicle may be used as a prognostic tool in the case of cicatricial alopecia. Anti-S100 antibody recognizes epidermal melanocytes and Langerhans cells (LCs), as well as dermal histiocytes. LCs (Langerin + , $\mathrm{CD} 1 \mathrm{a}+$ ) constituting subsets of DCs populating epidermis have been explored extensively along with interstitial DCs, namely, resident dermal myeloid DCs $(\mathrm{CD} 11 \mathrm{c}+, \mathrm{CD} 1 \mathrm{c}+)$, plasmacytoid blood DCs $(\mathrm{BDCA}-2+, \mathrm{CD} 123+)$, and a dermal population of CD14+ CD11c+ DCs found in normal skin, by other authors, who suggested that inflammatory skin diseases are characterized by a particular DC profile $[70,71]$. Over time, the spectra of effects of LCs on peripheral $\mathrm{T}$ cells, activation of skin resident memory $T$ lymphocytes, a defense function against pathogens and tissue repair were explored $[72,73]$. In our study, immunohistochemical results of S100 staining observed in the case of classic LPP and LP, both intraepithelial LCs and dermal DCs, are consistent with the results of Santoro et al. [49]. However, significant differences between the groups were not found by us, and this might indicate the similarity existing between clinical forms of LP. A low number of epidermal S100 positive cells found in psoriatic skin possibly indictes the status reflecting the remission stage of the disease.

Analysis of the literature data on the TUNEL evaluations suggests that a higher number of apoptotic keratinocytes was demonstrated in LP compared with healthy controls, but lower when LP is compared with other inflammatory diseases (psoriasis and cutaneous lupus erythematosus) $[74,75,76]$. Our findings regarding psoriatic skin samples studied 
are in accordance with the aforementioned literature data suggesting that psoriasis is characterized by aberrant differentiation and arrest of natural apoptotic process; to some extent, TUNEL positivity can be associated with hyperproliferation, in which actively replicating DNA is more abundant or accessible to detection.

Using the TUNEL assay and AI counting, we observed an increased number of apoptotic keratinocytes in classic LPP compared with LP. TUNEL reaction findings obtained by us are in accordance with evidence demonstrated by Bloor et al. and confirmed by Harries et al. reporting on increase of TUNEL positive epithelial cells in LP $\{14,24,40\}$. The results of TUNEL reaction supported by AI counting and ultrastructural analysis performed suggest that among patient groups analyzed in this study the basal and suprabasal keratinocyte damage was especially pronounced in the LPP of the scalp region, thus with high probability explaining hair loss. Without denying the fact that keratinocyte death is mediated by cytotoxic factors released, we made attempts to reach a goal of the study accentuating the significance of occurring nonspecific responses via damage of the basement membrane supporting keratinocytes, cellular cytoskeleton changes, and loss of intercellular junctions.

In conclusion, our study specified eHFSC death in cutaneous LP evidenced by CK15 immunohistochemistry and TUNEL reaction evaluations, and accentuated the role of cytoskeleton-dependent injury manifested as loss of intercellular contacts in eradication of damaged keratinocytes.

Riga Stradins University Department of Doctoral studies is acknowledged for reagents and publishing support.

The authors declare no conflict of interest.

\section{References}

1. Wolff K, Goldsmith L, Katz S, et al. Fitzpatrick's Dermatology in general medicine.7th ed. McGraw-Hill, New York 2008; 244-255.

2. Gorouhi F, Davari P, Fazel N. Cutaneous and mucosal lichen planus: a comprehensive review of clinical subtypes, risk factors, diagnosis, and prognosis. Scientific World Journal 2014; 2014: 742826

3. Sharma A, Bialynicki-Birula R, Schwartz RA, et al. Lichen planus: an update and review. Cutis 2012; 90: 17-23.

4. Arora SK, Chhabra S, Saikia UN, et al. Lichen planus: A clinical and immuno-histological analysis. Indian J Dermatol 2014; 59: 257-261.

5. Ross EK, Tan E, Shapiro J. Update on primary cicatricial alopecias. J Am Acad Dermatol 2005; 53: 1-37.

6. Cevasco NC, Bergfeld WF, Remzi BK, et al. A case-series of 29 patients with lichen planopilaris: The Cleveland Clinic Foun- dation experience on evaluation, diagnosis, and treatment. J Am Acad Dermatol 2007; 57: 47-53.

7. Rongioletti F, Christana K. Cicatricial (scarring) alopecias: an overview of pathogenesis, classification, diagnosis, and treatment. Am J Clin Dermatol 2012; 13: 247-260.

8. Assouly F, Reygagne P. Lichen Planopilaris: Update on Diagnosis and Treatment. Semin Cutan Med Surg 2009; 28: 3-10.

9. Colledge N, Walker B, Ralston S, et al. Davidson's principles and practice of medicine. $21^{\text {st }} \mathrm{ed}$. Churchill Livingstone/Elsevier, New York 2010; 1265-1266.

10. Freedberg I, Eisen A, Wolff K, et al. Fitzpatrick's dermatology in general medicine. $6^{\text {th }}$ ed. McGraw-Hill, New York 2003; 466.

11. Bardazzi F, Landi C, Orlandi C, et al. Graham Little-Piccardi-Lasseur syndrome following HBV vaccination. Acta Derm Venereol 1999; 79: 93.

12. Peters EM, Botchkarev VA, Botchkareva NV, et al. Hair-cycle-associated remodeling of the peptidergic innervation of murine skin, and hair growth modulation by neuropeptides. J Invest Dermatol 2001; 116: 236-245.

13. Peters EM, Liotiri S, Bodo E, et al. Probing the effects of stress mediators on the human hair follicle: substance $\mathrm{P}$ holds central position. Am J Pathol 2007; 171: 1872-1886.

14. Harries MJ, Paus R. The pathogenesis of primary cicatricial alopecias. Am J Pathol 2010; 177: 2152-2162.

15. Paus R, Cotsarelis G. The biology of hair follicles. N Engl J Med 1999; 341: 491-497.

16. Sabeti S, Malekzad F, Ashayer M, et al. The Rate and Pattern of $\mathrm{Bcl}-2$ and Cytokeratin 15 expression in trichoepithelioma and nodular basal cell carcinoma: a comparative study. Indian J Dermatol 2013; 58: 331-336.

17. Abbas O, Bhawan J. Expression of stem cell markers nestin and cytokeratin 15 and 19 in cutaneous malignancies. J Eur Acad Dermatol Venerol 2011; 25: 311-316.

18. Abbas O, Mahalingam M. Epidermal stem cells: practical perspectives and potential uses. Br J Dermatol 2009; 161: 228236.

19. Kloepper JE, Tiede S, Brinckmann J. Immunophenotyping of the human bulge region: the quest to define useful in situ markers for human epithelial hair follicle stem cells and their niche. Exp Dermatol 2008; 17: 592-609.

20. Ito M, Liu Y, Yang Z, et al. Stem cells in the hair follicle bulge contribute to wound repair but not to homeostasis of the epidermis. Nat Med 2005; 11: 1351-1354.

21. Cotsarelis G. Gene expression profiling gets to the root of human hair follicle stem cells. J Clin Invest 2006; 116: 19-22.

22. Al-Refu K. Stem cells and alopecia: a review of pathogenesis. Br J Dermatol 2012; 167: 479-484.

23. Mobini N, Tam S, Kamino H. Possible role of the bulge region in the pathogenesis of inflammatory scarring alopecia: lichen planopilaris as the prototype. J Cutan Pathol 2005; 32: 675679.

24. Harries MJ, Meyer K, Chaudhry I, et al. Lichen planopilaris is characterized by immune privilege collapse of the hair follicle's epithelial stem cell niche. J Pathol 2013; 231: 236-247.

25. Hoang MP, Keady M, Mahalingam M. Stem cell markers (cytokeratin $15, \mathrm{CD} 34$ and nestin) in primary scarring and nonscarring alopecia. Br J Dermatol 2009; 160: 609-615.

26. Pozdnyakova $\mathrm{O}$, Mahalingam M. Involvement of the bulge region in primary scarring alopecia. J Cutan Pathol 2008; 35: 922-925.

27. Habashi-Daniel A, Roberts JL, Desai N, et al. Absence of catagen/telogen phase and loss of cytokeratin 15 expression in hair follicles in lichen planopilaris. J Am Acad Dermatol 2014; 71: 969-972. 
28. Drogoszewska B, Chomik P, Polcyn A, et al. Clinical diagnosis of oral erosive lichen planus by direct oral microscopy. Postepy Dermatol Alergol 2014; 31: 222-228.

29. Sugerman PB, Savage NV, Walsh LV, et al. The pathogenesis of oral lichen planus. Crit Rev Oral Med 2012; 13: 350-365.

30. Saleh N, Samir N, Megahed H, Farid E. Homocysteine and other cardiovascular risk factors in patients with lichen planus. J Eur Acad Dermatol Venerol 2014; 28: 1507-1513.

31. Kastelan M, Massari LP. Focus on cell apoptosis research. Nova Publishers, New York 2007; 233-249.

32. Gaber MA, Maraee AH, Alsheraky DR, Azeem MH. Immunohistochemical expression of perforin in lichen planus lesions. Ultrastruct Pathol 2014; 38: 413-419.

33. Su SC, Chung WH. Cytotoxic proteins and therapeutic targets in severe cutaneous adverse reactions. Toxins (Basel) 2014; 6: 194-210.

34. Neppelberg E, Johannessen AC, Jonsson R. Apoptosis in oral lichen planus. Eur J Oral Sci 2001; 109: 361-364.

35. Ernst N, Yay A, Bíró T, et al. $\beta 1$ integrin signaling maintains human epithelial progenitor cell Survival in situ and controls proliferation, apoptosis and migration of their progeny. PLoS One 2013; 8: e84356.

36. Chularojanamontri L, Tuchinda P, Triwongwaranat D, et al. Diagnostic significance of colloid body deposition in direct immunofluorescence. Indian J Dermatol Venereol Leprol 2010; 76: 373-377.

37. Burgdorf WH, Plewig G. Who described civatte bodies? J Cutan Pathol 2014; 41: 340-346.

38. Masu S, Sato A, Seiji M. Electron microscopic studies on Civatte body in Riehl's melanosis. Tohoku J Exp Med 1980; 131: 177-196.

39. Vij H, Vij R, Rao NN, et al. Quantification of colloid bodies in oral lichen planus and oral lichenoid reaction - a histochemical study. J Clin Exp Dent 2011; 3: 207-211.

40. Bloor BK, Malik FK, Odell EW, et al. Quantitative assessment of apoptosis in oral lichen planus. Oral Surg 1999; 88: 187. 195.

41. Tanda N, Mori S, Saito K, et al. Expression of apoptotic signalling proteins in leukoplakia and oral lichen planus: quantitative and topographical studies. J Oral Pathol Med 2000; 29: 385-393.

42. Bascones-Ilundain C, Gonzalez-Moles MA, Esparza G, et al. Significance of liquefaction degeneration in oral lichen planus: a study of its relationship with apoptosis and cell cycle arrest markers. Exp Dermatol 2007; 32: 556-563.

43. Fritz G, Hugo M, Botelho MH, et al. Natural and amyloid self-assembly of S100 proteins: structural basis of functional diversity. FEBS Journal 2010; 277: 4578-4590.

44. Lukanidin E, Sleeman JP. Building the niche: the role of the S100 proteins in metastatic growth. Sem Cancer Biol 2012; 22: 216-225.

45. Halawi A, Abbas O, Mahalingam M. S100 proteins and the skin: a review. J Eur Acad Dermatol Venereol 2014; 28: 405414.

46. Takaishi M, Makino T, Morohashi M, Huh NH. Identification of human hornerin and its expression in regenerating and psoriatic skin. J Biol Chem 2005; 280: 4696-4703.

47. Fleming JM, Ginsburg E, Oliver SD, et al. Hornerin, an S100 family protein, is functional in breast cells and aberrantly expressed in breast cancer. BMC Cancer 2012; 12: 266.

48. Eckert RL, Broome AM, Ruse M, et al. S100 Proteins in the epidermis. J Invest Dermatol 2004; 123: 23-33.

49. Santoro A, Majorana A, Roversi L, et al. Recruitment of dendritic cells in oral lichen planus. J Pathol 2005; 205: 426-434.

50. Broome AM, Ryan D, Eckert RL. S100 protein subcellular localization during epidermal differentiation and psoriasis. J Histochem Cytochem 2003; 51: 675-685.
51. Schonthaler HB, Guinea-Viniegra J, Wculek SK, et al. S100A8-S100A9 protein complex mediates psoriasis by regulating the expression of complement factor C3. Immunity 2013; 39: 1171-1181.

52. Lee MS, Wilkinson B, Doyle JA, et al. A comparative immunohistochemical study of lichen planus and discoid lupus erythematosus. Australas J Dermatol 1996; 57: 188-192.

53. Wenzel J, Peters B, Zahn S, et al. Gene expression profiling of lichen planus reflects CXCL9+-mediated inflammation and distinguishes this disease from atopic dermatitis and psoriasis. J Invest Dermatol 2008; 128: 67-78.

54. Brant JMC, Vasconcelos AC, Rodrigues LV. Role of apoptosis in erosive and reticular oral lichen planus exhibiting variable epithelial thickness. Braz Dent J 2008; 19: 179-185.

55. McKee PH. Essential skin pathology. Mosby International Ltd, Hong Kong 1999; 78.

56. Poblet E, Jimenez F, Pascual A, et al. Frontal fibrosing alopecia versus lichen planopilaris: a clininicopathological study. Int J Dermatol 2006; 45: 375-380.

57. Mirmirani P, Willey A, Headington JT, et al. Primary cicatricial alopecia: histopathologic findings do not distinguish clinical variants. J Am Acad Dermatol 2005; 52: 637-643.

58. Olsen EA, Bergfeld WF, Cotsarelis G, et al. Summary of North American Hair Research Society (NAHRS) - Sponsored workshop on cicatricial alopecia, Duke University Medical Center, February 10 and 11, 2001. J Am Acad Dermatol 2003; 48: 103-110.

59. Jiang S, Zhao L, Purandare B, Hantash BM. Differential expression of stem cell markers in human follicular bulge and interfollicular epidermal compartments. Histochem Cell Biol 2010; 133: 455-465.

60. Inoue K, Aoi N, Sato T, et al. Differential expression of stem-cell-associated markers in human hair follicle epithelial cells. Lab Invest 2009; 89: 844-856.

61. Gao Y, Li M, Zhang X, et al. Isolation, culture and phenotypic characterization of human sweat gland epithelial cells. Int J Mol Med 2014; 34: 997-1003.

62. Misago N, Takai T, Toda S, et al. The changes in the expression levels of follicular markers in keratoacanthoma depend on the stage: keratoacanthoma is a follicular neoplasm exhibiting infundibular/isthmic differentiation without expression of CK15. J Cutan Pathol 2014; 41: 437-446.

63. Al-Refu K, Edward S, Ingham E, Goodfield M. Expression of hair follicle stem cells detected by cytokeratin 15 stain: implications for pathogenesis of the scarring process in cutaneous lupus erythematosus. Br J Dermatol 2009; 160: 1188-1196.

64. Taylor G, Lehrer MS, Jensen PJ, et al. Involvement of follicular stem cells in forming not only the follicle but also the epidermis. Cell 2000; 102: 451-461.

65. Wilbur DC, Brachtel EF, Gilbertson JR, et al. Whole slide imaging for human epidermal growth factor receptor $2 \mathrm{im}$ munohistochemistry interpretation: Accuracy, Precision, and reproducibility studies for digital manual and paired glass slide manual interpretation. J Pathol Inform 2015; 6: 22.

66. Swiderska Z, Korzynska A, Markiewicz T, et al. Comparison of the manual, semiautomatic, and automatic selection and leveling of hot spots in whole slide images for Ki-67 quantification in meningiomas. Anal Cell Pathol (Amst) 2015; 2015: 498746.

67. Gavrielides MA, Conway C, O'Flaherty N, et al. Observer performance in the use of digital and optical microscopy for the interpretation of tissue-based biomarkers. Anal Cell Pathol (Amst) 2014; 2014: 157308.

68. Yagi Y, Riedlinger G, Xu X, et al. Development of a database system and image viewer to assist in the correlation of histopathologic features and digital image analysis with clinical and molecular genetic information. Pathol Int 2016; 66: 63-74. 
69. Castellana D, Paus R, Perez-Moreno M. Macrophages contribute to the cyclic activation of adult hair follicle stem cells. PLoS Biol 2014; 12 (12): e1002002.

70. Johnson-Huang LM, McNutt NS, Krueger JG, Lowes MA. Cytokine-producing dendritic cells in the pathogenesis of inflammatory skin diseases. J Clin Immunol 2009; 29: 247-256.

71. Steinman RM. Decisions about dendritic cells: past, present, and future. Annu Rev Immunol 2012; 30: 1-22.

72. Romani N, Clausen BE, Stoitzner P. Langerhans cells and more: langerin-expressing dendritic cell subsets in the skin. Immunol Rev 2010; 234: 120-141.

73. Klechevsky E. Human dendritic cells - stars in the skin. Eur J Immunol 2013; 43: 3147-3155.

74. Gündüz K, Demireli P, Vatansever S, et al. Examination of bcl2 and $\mathrm{p} 53$ expressions and apoptotic index by TUNEL method in psoriasis. J Cutan Pathol 2006; 33: 788-792.

75. Kawashimaa K, Doib H, Itoa Y, et al. Evaluation of cell death and proliferation in psoriatic epidermis. J Dermatol Sci 2004 35: 207-214.

76. Toberer F, Sykora J, Göttel D, et al. Apoptotic signal molecules in skin biopsies of cutaneous lupus erythematosus: analysis using tissue microarray. Exp Dermatol 2013; 22: 656-681.

\section{Address for correspondence}

\section{Ilze Upeniece}

Department of Infectology and Dermatology,

Riga Stradins University

Valdeku St. 17-35

LV-1004 Riga, Latvia

e-mail: dr.ilzeupeniece@gmail.com 